\title{
Local Structural Analysis: a Primer
}

\author{
Delalandre Mathieu ${ }^{1}$, Trupin Eric ${ }^{1}$, Ogier Jean-Marc ${ }^{2}$ \\ ${ }^{1}$ PSI Laboratory, University of Rouen, 76821 Mont Saint Aignan, France \\ E-mail: \{mathieu.delalandre ; eric.trupin\}@univ-rouen.fr \\ ${ }^{2}$ L3I Laboratory, University of La Rochelle, 17042 La Rochelle, France \\ E-mail:jmogier@univ-lr.fr
}

\begin{abstract}
The structural analysis is a processing step during which graphs are extracted from binary images. We can decompose the structural analysis into local and global approaches. The local approach decomposes the connected components, and the global approach groups them together. This paper deals especially with the local structural analysis. The local structural analysis is employed for different applications like symbol recognition, line drawing interpretation, and character recognition. We propose here a primer on the local structural analysis. First, we propose a general decomposition of the local structural analysis into four steps: object graph extraction, mathematical approximation, high-level object construction, and object graph correction. Then, we present some considerations on the method comparison and combination.
\end{abstract}

Keywords: shape analysis, graph construction, graphics document, symbol, handwriting

\section{Introduction}

The problem of document image interpretation is a vast field gathering three main applications: handwriting [58], graphics documents (technical documents [51], maps [43], symbols [32], and so on.), and structured documents [39]. Document image interpretation is an artificial intelligence problem based on three entities: a control system, a pattern recognition process for document images ${ }^{1}$, and a knowledge base. Several common works on this problem have been realized during the last fifteen years [12].

This paper deals with the pattern recognition process. Classically, a pattern recognition process is decomposed into two main steps [25]. The first one is an image processing step which has two goals: the image pre-processing allowing to enhance the image's conditions, and the feature extraction for the description of image's shapes. In the following of this paper, as [34], we simply call the feature extraction step "analysis". The second one is the recognition step. This step exploits the extracted features by the analysis step for different purposes like: recognition [33], learning [37], indexing [15], data structuring [59], interest zone search [13], and so on. Two main approaches for the pattern recognition exist: structural \& syntactic $^{2}$ [53], and statistical \& connexionnist [24]. This paper deals especially with the structural approach. In this approach, the image-processing step extracts graphs from images and the recognition step exploits them. Many different shapes could be described by graphs in document image interpretation such as: forms [39], oriental characters [18], and graphics parts [51].

The structural recognition step is a graph exploitation problem, which uses two main approaches: graphmatching [19] and grammar [4]. The first one matches extracted graphs with model graphs. The second one applies different rules to transform extracted graphs into model graphs. A graph problem depends on two criteria: graph/subgraph, and exact-inexact. A subgraph is a subset of node and edge of a larger graph. The subgraph problem is to recognize a model subgraph into a candidate graph. If extracted graphs correspond exactly to model graphs, the problem is known as exact. Unfortunately, in image applications, graphics parts are often connected to other parts, and extracted graphs are noisy and large sized. So, it is an inexact subgraph problem, into candidate graphs large sized.

\footnotetext{
${ }^{1}$ In the following, we talk about "recognition process" for "recognition process for document images".

${ }^{2}$ In the following, we talk about "structural" for "structural \& syntactic".
} 
The analysis step extracts (or constructs) graphs from images. For the purpose of this paper, we simply call it "structural analysis". We can decompose the structural analysis into local and global approaches ${ }^{3}$. The boundary between these two approaches is the connected component. The local approach decomposes a connected component into basic object, and the global approach groups together connected components according to some closeness and connection constraints. The Figure 1 gives an example: the local approach (b) decomposes the connected component (a) into arc, junction, and vector objects; the global approach (c) groups together three connected components (a) according to some closeness and connection constraints.

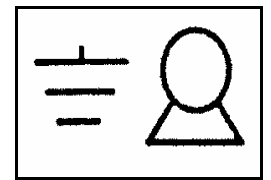

(a)

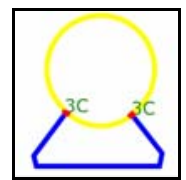

(b)

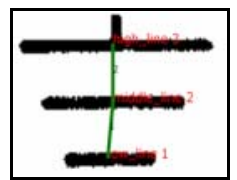

(c)

Figure 1: global and local approaches

This paper especially deals with the local structural analysis. We propose here a primer on the local structural analysis. In section 2, we give a general decomposition of the local structural analysis into four steps. Then, in section 3, we present a comparison study of methods. In section 4, we present some considerations on the method combination. Finally in section 5, we conclude.

\section{General Decomposition}

The local analysis employs different approaches from character recognition (latin [55], oriental [18]), and graphics recognition (technical documents [51], maps [43], symbols [32], and so on.). It extracts different objects from documents according to the exploration granularity. The smallest objects are the pixels and the biggest objects are the connected components. We propose here a general decomposition of the local structural analysis into four steps: object graph extraction, mathematical approximation, high-level object construction, and object graph correction. We present each step in sections 2.1, 2.2, 2.3, and 2.4.

\subsection{Object Graph Extraction}

This step decomposes the connected component into an object graph. We have listed seven method families as shown in the Figure 2. The methods are based on: skeletonisation (a), contouring (b), tracking (c), run decomposition (d), region decomposition (e), mesh decomposition (f), and object segmentation (g). We present each method family in the next sections.

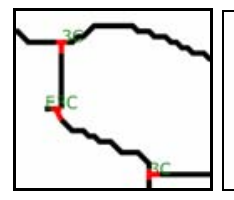

(a)

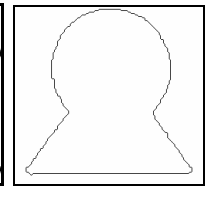

(b)

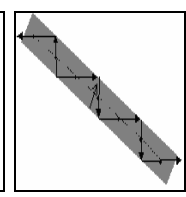

(c)

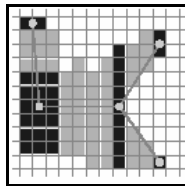

(d)

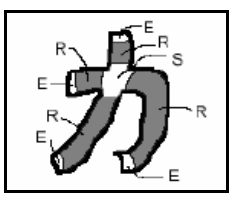

(e)

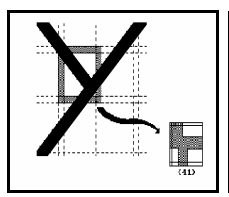

(f)

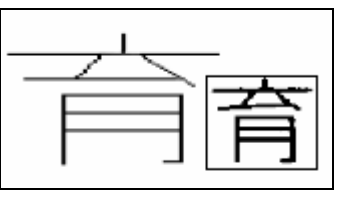

$(\mathrm{g})$

Figure 2 : (a) skeletonisation (b) contouring (c) tracking (d) run decomposition (e) region decomposition (f) mesh decomposition (g) object segmentation

\footnotetext{
${ }^{3}$ This classification also exists in statistical analysis [41].
} 


\subsubsection{Skeletonisation Based Methods}

The skeletonisation based methods are the most commonly used. They involve two steps. The first one extracts the skeleton images [26]. Two main families exist, by distance transform and by iterative thinning [1]. The second one analyses skeleton images in order to extract pixel graphs. It uses different methods, based on the connectivity analysis [54] [44], or the 3-connected pixel destruction [14] [27]. The Figure 2 (a) gives an example of an extracted pixel graph [14].

\subsubsection{Contouring Based Methods}

The contouring based methods are often used. Two method families exist [56]. The first methods use contour images like intermediate representations. They are based on mathematical morphology [20] or on neighbouring tests [14]. Similar to skeletonisation based methods, they involve a second step to extract the contours' pixel chains. The second methods directly extract the contours' pixel chains without any intermediate image representation. They use line following methods [1], or blob coloring methods [14]. Their advantages rely on the fact that they provide the inclusion relations between chains, and they permit the selection of internal or external contours [14]. The Figure 2 (b) gives an example of external contours' pixel chains of the Figure 1 [14].

\subsubsection{Tracking Based Methods}

The tracking based methods directly analyse the images without any intermediate representation. They are based on the structuring elements use in order to track the shapes, of pixel type [16] [48], or area type (circle [11], gaussian bead [61]). They produce geometric object graphs (circle and vector [48]), or pixel graphs [11]. They depend on the adopted tracking model (linear [16], circular [48]), and on the structuring element's progression into the shape (continuous [11], by jump [16]). The tracking process may be of two types: line tracking and junction tracking. In both cases, the employed structuring element can be of "pixel type" [11] [16], of "area type" [11] [61], or even both [42]. The Figure 2 (c) gives an example of pixel tracking [16].

\subsubsection{Run Decomposition Based Methods}

The run decomposition based methods are used for line drawing interpretation [6] and handwriting recognition [17] [60]. A run is a maximal sequence of black pixel in a column or a row of the image. The run graph is constructed with vertical and horizontal runs according to construction rules. From this definition, [6] constructs the "Mixed Run Graph", a vertical and horizontal run graph (Figure 2 (d)). During this construction, vertical and horizontal runs are merged into junction nodes and line nodes in the final graph.

\subsubsection{Region Decomposition Based Methods}

The region decomposition based methods are less used in the literature. They decompose a connected component into different regions. [7] [9] compute orientations data of each image's pixel with its contour pixels. Then, they search the majority directions for each pixel, and construct like this the line, extremity, and junction regions (Figure 2 (e)). In [14], we propose region decomposition method based on a wave aggregation. The wave breaking and stopping cases define the regions' boundaries. In the following step, the region graph is analysed to construct the line and junction regions.

\subsubsection{Meshes Decomposition Based Methods}

The mesh decomposition based methods have been used for vectorisation applications [29] [57]. Image is firstly split up into meshes. Then, the meshes are recognized according to a mesh library (Figure 2 (f)) [57]. So, the result mesh map is analysed to construct the structural relations between the meshes. 


\subsubsection{Segmented Object Based Methods}

The segmented object based methods are often used in vision [38] and in document image interpretation [52]. The segmented object methods directly extract the objects such as lines, arcs, ellipsis, and junctions. These methods employ mathematical transforms in order to change the image's representation space. This representation space is used to find the objects according to their mathematical models. [52] extracts like this the vertical, diagonal, and horizontal lines for Chinese handwriting recognition (Figure 2 (g)). Some works deal with the junction segmentation [10] (' $T$ ' junction, ' $\mathrm{X}$ ' junction, and so on.). Different techniques exist like the Hough transform [38] or the Gabor filters [10]. If the system does not deal with the junction segmentation, the mathematical objects' crossings [36] and the connections between mathematical objects' extremities [52] are searched to construct the structural relations.

\subsection{Mathematical Approximation}

During this step, the step 1's result objects (section 2.1) are approximated by mathematical objects like vectors, arcs, elliptical arcs, and curves (Figure 1 (a)). The mathematical approximation functions can exploit various entry data like: pixels, vectors, curves, and circles. In fact, we can approximate vectors into circles, curves into circles, and so on. The pixel and vector graphs (Figure 2 (a), (b), (c)) are often used. The region and run graphs (Figure 2 (d), (e)) are also used after their skeletons and contours extractions [17] [14]. The mesh and segmented object graphs (Figure 2 (f), (g)) are not used because their objects are enough approximated. So in practice, graphs and chains of pixel are the most commonly used for the vectorisation step [31] [54]. An algorithm permitting a combination of mathematical object approximation and an overview can be found in [46]. Also, an algorithm to extract contextual information on the data quality (in order to control a system in the approximation algorithm choice) can be found in [47].

\subsection{High-Level Object Construction}

After the mathematical approximation step, some systems construct from low-level objects (vectors, arcs, curves) higher level objects like circles, parallelograms, triangles, and so on. These objects are constructed from skeletonisation based process [23] or contouring based process [28] [8] [62] (we talk about contour matching). The contour matching is generally used to rebuild the shapes' junctions [28]. Position constraints of mathematical objects are tested during this high-level object construction. So, this construction is not only a graph factorisation step. After this construction, new structural representations can be created, describing the structural relations between the high-level objects [45]. The Figure 3 (a) gives a use-case for circle reconstruction from skeletonisation based process [23]. The Figure 3 (b) gives an example of contour matching into triangle graph [62].

\subsection{Object Graph Correction}

Some systems analyse the extracted object graphs to correct the structural descriptions. [50] distinguishes systems with corrections (two steps) and without correction (one step). Some corrections add or delete some nodes and edges into object graphs. Other corrections compute new edges or nodes' attributes of graphs. These correction processings use image processing steps, so they are not only graph factorisation processings. These corrections can be used on different data type in (or between) each of steps 1, 2, and 3 (sections 2.1, 2.2, and 2.3).

On the pixel graphs different correction kind can be used as: pruning and merging [14] (Figure 3 (c)), and the correction of junctions' distortions [30] (Figure 3 (d)). These correction methods can be also used on vector graphs [16], but in this case the junction correction by vector crossing search is also used [22] (Figure 3 (e)). [57] corrects its mesh graphs by a decomposition/fusion processing. [17] corrects its run graphs with fusion/suppression processings of segmented/isolated runs. [45] corrects its high-level object graphs with a merging processing. 


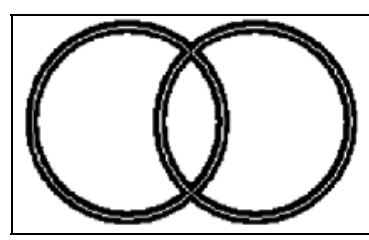

(a)

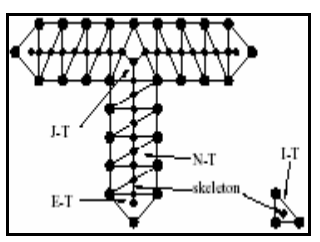

(b)

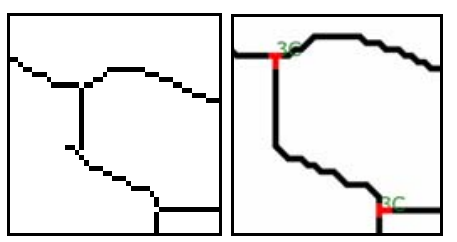

(c)

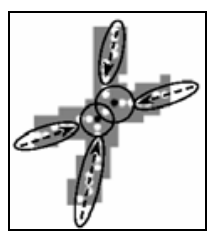

(d)

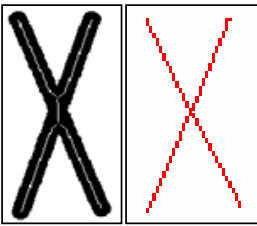

(e)

Figure 3 : (a) circle construction case (b) contour matching

(c) (d) skeleton correction (e) vectorial correction

\section{Method Comparison}

We compare in the Table 1 the advantages and drawbacks of the object graph extraction (section 2.1). The comparisons concerning mathematical approximation step's methods (section 2.2), high-level object construction step's methods (section 2.3), and object graph correction step's methods (section 2.4), that are not detailed in this paper and can be found in [46], [40], and [5]. We compare the object graph extraction methods according to seven criteria which are respectively named "Junction", "Morphology", "Invariance", "Sensitivity", "Semantic", "Reversibility", and "Complexity". "Junction" criterion specifies the method's ability to detect the shapes' junctions. "Morphology" criterion specifies the method's ability to analyse heterogeneous shapes. "Invariance" criterion specifies the method's ability to analyse multiple scales and orientations of shapes. "Sensitivity" criterion specifies the method's noise resistance. "Semantic" criterion specifies the method's information adding for the shapes' descriptions. "Reversibility" criterion specifies the method's ability to restore the raster data. "Complexity" criterion specifies the method's algorithmic complexity. This comparison study is only based on a set of significant experiments performed in our laboratories.

\begin{tabular}{|l|c|c|}
\cline { 2 - 3 } \multicolumn{1}{c|}{} & Advantages & Drawbacks \\
\hline Skeleton & Invariance & Morphology, Sensitivity, Complexity \\
\hline Contouring & Morphology, Invariance, Reversibility & Junction \\
\hline Tracking & Junction, Semantic, Complexity & Morphology \\
\hline Run & Junction, Morphology, Reversibility & Invariance, Complexity \\
\hline Region & Junction, Morphology, Reversibility & Semantic, Complexity \\
\hline Mesh & Junction, Semantic, Complexity & Morphology, Invariance, Sensitivity, Reversibility \\
\hline Segmentation & Sensitivity, Semantic & Junction, Morphology, Invariance, Reversibility, Complexity \\
\hline
\end{tabular}

Table 1: method comparison

The skeletonisation based methods are invariant [54], but they only permit the linear shape analysis [45], and are noise sensitive (especially for the junction zone analysis [54]).

The contouring based methods permit to analyse all the shape types, and are reversible [20]. Their drawback is the non detection of junctions that must rebuilt with a high-level object construction step [28].

The tracking based methods permit a good junction detection. Beside, they export vectorial data, and are of low complexity [49]. However, they have some difficulties with shapes' thickness variation [42].

The run decomposition based methods [6] permit a good junction detection, any shape type analysis, and a raster restoration. However, they are orientation sensitive (because of vertical and horizontal run types) and complex (because of runs encoding and structuring). 
The region decomposition based methods also permit a good junction detection, allow to analyse all the shape types, and permit a raster restoration. However, the regions give few information like representation object (analysis must be completed [13] [14]), and have a high memory cost of manipulation.

The mesh decomposition based methods permit a good junction detection, a complex object export, and are of low complexity. However, these methods are very sensitive to the initial positions of considered meshes [57] (invariance, sensitivity) and strongly depend on mesh library / shapes types adequacy.

The segmented object based methods are noise resistant [50], and export geometrical objects [50]. However, the mathematical transforms used are complex [38], based on the known model search [50] (reversible, morphology), limited to some orientations [52] (invariance), and have some difficulties with the junction segmentation [10].

\section{Method Combination}

Several research perspectives exist on the subject dealing with the local structural analysis. Some works use recognition step in order to control the graphs' constructions with knowledge bases. These controls can be used for all analysis levels and approaches (sections 2.1, 2.2, 2.3, and 2.4) [2] [52] [59] [6]. Other works deal with the segmentation/recognition problem by utilization of "system approaches", like: the perceptive approaches [61] for instance, or multi-agent approaches [21]. Finally, some works use strategic approaches in order to combine the methods [14]. We develop this last perspective in this section.

We propose here a combinations' classification into three categories: comparative, hybrid, and cooperative. These combinations are essentially local, but some of them deal with the local/global aspects [13] [3]. The comparative combinations analyze the shapes in order to extract different graphs from different methods. These graphs are then compared during the recognition step. [40] compares vector graphs obtained from contour and skeleton images. In [13] we compare loop graphs (global) with skeleton graphs (local). The hybrid combinations analyze the shapes in order to extract hybrid graphs. These graphs are the combinations of two (or several) analysis methods. Besides, the global methods [13] and local [6] [7] [14] exploiting the region objects permit to use a statistico-structural approach [13] [14]. [3] extracts connected component graphs, and completes these graphs with the concavity local information for each connected component. In [13] and [14] we use statistico-structural approaches for the recognition of local/global region graphs. The cooperative combinations exploit the analysis methods in order to simplify the recognition process's complexity. [49] uses an object progressive simplification process. In [13] we simplify our global analysis by the use of a local analysis.

The comparative and hybrid combinations permit the multi-models representations of shapes (or adopted graph model). The multi-model representation's possibilities are obtained by the combinations local, local/global, and statistico-structural. The works on the construction and exploitation of the multi-model representation certainly constitute an important research perspective of the local structural analysis.

\section{Conclusion}

In this paper, we propose a primer on the local structural analysis. This analysis decomposes the connected components into graph of basic object. Then, these graphs are exploited during the recognition step. This analysis declines itself according to different construction levels, using different methods. Each method presents some advantages and drawbacks. All these methods can be combined, for especially the multimodel representation. These multi-models representations certainly constitute an important research perspective of the local structural analysis.

\section{Acknowledgement}

The authors wish to thank Noorazrim Zakaria (L3I Laboratory, La Rochelle University, France) and JeanYves Ramel (LI Laboratory, Tours University, France) for their contribution to this work. 


\section{$7 \quad$ References}

[1] S. Ablameyko, T.P. Pridmore, "Machine Interpretation of Line Drawing Images", Springer-Verlag, 2000.

[2] M. Ahmed, R. Ward, "A Rotation Invariant Rule Based Thinning Algorithm for Character Recognition", Pattern Analysis and Machine Intelligence (PAMI), 24(12) : 1672-1678, 2002.

[3] O. El Badawy, M. Kamel, "Shape Representation using Concavity Graphs", International Conference on Pattern Recognition (ICPR), 2002.

[4] D. Blostein, H. Fahmy, A. Grbavec, "Issues in the Practical Use of Graph Rewriting", Graphics Recognition (GREC), 1995.

[5] E. Bodansky, A. Gribov, M. Pilouk, "Post-processing of Lines Obtained by Raster-to-Vector Conversion", Graphics Recognition (GREC), 2001.

[6] M. Burge, W.G. Kropatsh, "A Minimal Line Property Preserving Representation of Line Images", Structural and Syntactical Pattern Recognition (SSPR), 1998.

[7] R. Cao, C.L. Tan, "A Model of Stroke Extraction from Chinese Character Images", International Conference on Pattern Recognition (ICPR), 2000.

[8] F. Chang, Y.C. Lu, T. Palvidis, "Feature Analysis Using Line Sweep Thinning Algorithm", Pattern Analysis and Machine Intelligence (PAMI), 21(2) : 145-158, 1999.

[9] Y.S. Chen, "Segmentation and Association Among Lines and Junctions for a Line Image", Pattern Recognition (PR), 27(9) : 1135-1157, 1994.

[10] J. Chen, Y. Sato, S. Tamura, "Orientation Space Filtering for Multiple Orientation Line Segmentation", Pattern Analysis and Machine Intelligence (PAMI), 22(5) : 417-429, 2000.

[11] J. Chiang, S. Tue, "A New Algorithm for Line Image Vectorization”, Pattern Recognition (PR), 31(10) : 1541-1549, 1998.

[12] D. Crevier, R. Lepage, "Knowledge-Based Image Understanding Systems: A Survey", Computer Vision and Image Understanding (CVIU), 67(2) : 161-185, 1997.

[13] M. Delalandre, S. Nicolas, E. Trupin, J.M. Ogier, "Symbols Recognition by Global-Local Structural Approaches, Based on the Scenarios Use, and with a XML Representation of Data", International Conference on Document Analysis And Recognition (ICDAR), 2003.

[14] M. Delalandre, Y. Saidali, J.M. Ogier, E. Trupin, "Adaptable Vectorisation System Based on Strategic Knowledge and XML Representation Use", Graphics Recognition (GREC), 2003.

[15] D. Doermann, "The Indexing and Retrieval Document, a Survey", Technical Report CS-TR-3876, University of Maryland Computer Science Department, USA, 1998.

[16] D. Dori, "Sparse Pixel Vectorisation: An Algorithm and its Performance Evaluation", Pattern Analysis and Machine Intelligence (PAMI), 21(3) : 202-215, 1999.

[17] K.C. Fan, W.H. Wu, "A Run Length Coding Based Approach to Stroke Extraction of Chinese Characters", Pattern Recognition (PR), 33(11) : 1881-1895, 2000.

[18] J. Fan, "Off-line Optical Character Recognition for Printed Chinese Character-A Survey", Technical Report, University of Colombia, USA, 2002.

[19] E. Hancock, R. Wilson, "Graph-Based Methods for Vision: A Yorkist Manifesto", Structural and Syntactical Pattern Recognition (SSPR), 2002.

[20] Y.M.Y Hasan, L.J Karan, "Morphological Reversible Contour Representation", Pattern Analysis and Machine Intelligence (PAMI), 22(3) : 227-239, 2000.

[21] T.C. Henderson, L. Swaminathan, "Agent Based Engineering Drawing Analysis", Symposium on Document Image Understanding Technology (SDIUT), 2003.

[22] X. Hilaire and K. Tombre, "Improving the Accuracy of Skeleton-Based Vectorisation", Graphics Recognition (GREC), 2001.

[23] X. Hilaire, "Ranvec and the Arc Segmentation Contest", Graphics Recognition (GREC), 2001. 
[24] A.K. Jain, R.P.W. Duin, J. Mao, "Statistical Pattern Recognition : a Review", Pattern Analysis and Machine Intelligence (PAMI), 22(1) : 4-37, 2000.

[25] R. Kasturi, L. O'Gorman, V. Govindaraju, "Document Image Analysis: A Primer”, Sadhana, 27(1) : 3-22, 2002.

[26] L. Lam, C.Y. Suen, “An Evaluation of Parallel Thinning Algorithms for Character Recognition”, Pattern Analysis and Machine Intelligence (PAMI), 17(9) : 914-919, 1995.

[27] K.K. Lau, P.C. Yuen, Y.Y. Tang, "Stroke Extraction and Stroke Sequence Estimation On Signatures", International Conference on Pattern Recognition (ICDAR), 2002.

[28] C. Lee, B. Wu, "A Chinese Character Stroke Extraction Algorithm Based on Contour Information", Pattern Recognition (PR), 31(6) : 651-653, 1998.

[29] X. Lin, S. Shimotsuji, M. Mihoh, T. Sakai, "Efficient Diagram Understanding with Characteristic Pattern Detection", Computer Vision Graphics and Image Processing, 30 : 84-106, 1985.

[30] F. Lin, X. Tang, "Off-line Handwritten Chinese Character Stroke Extraction", International Conference on Pattern Recognition (ICPR), 2002.

[31] W. Liu, D. Dori, "From Raster to Vectors : Extracting Visual Information from Line Drawings", Pattern Analysis and Applications (PAA), 2(2) : 10-21, 1999.

[32] J. Lladós, E. Valveny, G. Sánchez, E. Martí, "Symbol Recognition : Current Advances an Perspectives", Graphics Recognition (GREC), 2001.

[33] J. Llados, E. Marti, J.J. Villuanueva, "Symbol Recognition by Error Subgraph Matching Between Region Adjacency Graphs", Pattern Analysis and Machine Intelligence (PAMI), 23(10) : 1137-1143, 2001.

[34] S. Locarnic, "A Survey of Shape Analysis Techniques", Pattern Recognition (PR), 31(8) : 983-1001, 1998.

[35] P.K. Loo, C.L. Tan, "Detection of Word Group Based on Irregular Pyramid", International Conference on Document Analysis And Recognition (ICDAR), 2001.

[36] K. Loudon, "Mastering Algorithms with C", O'Reilly Editions, 2000.

[37] Y. Luo, W. Liu, "Engineering Drawings Recognition Using a Case-based Approach", International Conference on Document Analysis And Recognition (ICDAR), 2003.

[38] J. Matas, C. Galambos, J. Kittler, "Progressive Probabilistic Hough Transform for Line Detection", Computer Vision and Pattern Recognition (CVPR), 1999.

[39] G. Nagy, "Twenty Years of Document Image Analysis in PAMI", Pattern Analysis and Machine Intelligence (PAMI), 22(1) : 38-62, 2000.

[40] Y. Nakajima, S. Mori, S. Takegami, S. Sato, "Global Methods for Stroke Segmentation", International Journal Document Analysis and Recognition (IJDAR), 2 : 19-23, 1999.

[41] J. Neumann, H. Samet, A. Soffer, "Integration of Local and Global Shape Analysis for Logo Classification", International Workshop on Visual Form (IWVF), 2001.

[42] J.M. Ogier, C. Olivier, Y. Lecourtier, "Extraction of Roads from Digitized Maps", European Signal Processing Conference (EUSIPCO), 1992.

[43] J.M. Ogier, S. Adam, A. Bessaid, H. Bechar, "Automatic Topographic Color Map Analysis System", Graphics Recognition (GREC), 2001.

[44] D.V. Popel, "Compact Graph Model of Handwritten Images: Integration into Authentification and Recognition", Structural and Syntactical Pattern Recognition (SSPR), 2002.

[45] J.Y. Ramel, N. Vincent, H. Emptoz, "A Structural Representation for Understanding Line-Drawing Images", International Journal on Document Analysis And Recognition (IJDAR), 3 : 58-66, 2000.

[46] P.L. Rosin, A.W. West, "Nonparametric Segmentation of Curves Into Various Representations", Pattern Analysis and Machine Intelligence (PAMI), 17(12) : 1140-1153, 1995.

[47] P.L. Rosin, "Techniques for Assessing Polygonal Approximation of Curves", Pattern Analysis and Machine Intelligence (PAMI), 19(6) : 659-666, 1997. 
[48] J. Song, F. Su, C. Tai, S. Cai, "An Object-Oriented Progressive-Simplification based Vectorisation System for Engineering Drawings: Model, Algorithm and Performance", Pattern Analysis and Machine Intelligence (PAMI), 24(8) : 1048-1060, 2002.

[49] J. Song, M. Cai, M.R. Lyu, S. Cai, "Graphics Recognition from Binary Images : One Step or Two Steps", International Conference on Pattern Recognition (ICPR), 2002.

[50] J. Song, M. Cai, M.R. Lyu, S. Cai, "A New Approach for Line Recognition in Large-Size Images Using Hough Transform”, International Conference on Pattern Recognition (ICPR), 2002.

[51] J. Song, M.R. Lyu, M. Cai, and S. Cai, "Graphic Object Recognition from Binary Images: a Survey and an Integrated Paradigm", Transactions on Systems, Man and Cybernetics, part C: Applications and Reviews (TSMCC), under review.

[52] Y.M. Su, J.F Wang, "A Learning Process to the Identification of Feature Points on Chinese Characters", International Conference on Pattern Recognition (ICPR), 2002.

[53] K. Tombre, "Structural and Syntactic Methods in Line Drawing Analysis : To Which Extent do they Work? ”, Structural and Syntactical Pattern Recognition (SSPR), 1996.

[54] K. Tombre, C. Ah-Soon, P. Dosch, G. Masini, S.Tabbone, "Stable and Robust Vectorization : How to Make the Right Choices", Graphics Recognition (GREC), 1999.

[55] O. D. Trier, A. K. Jain, T. Taxt, "Features Extraction Methods for Character Recognition - A Survey", Pattern Recognition (PR), 29(4) : 641-662, 1996.

[56] M.J. Turner, N.E. Wiseman, "Efficient Lossless Image Contour Coding", Computer Graphics Forum, 15(2) : 107-118, 1996.

[57] P. Vaxivière, K. Tombre, "Subsampling : A Structural Approach to Technical Document Vectorisation", Syntactic and Structural Pattern Recognition (SSPR), 1995.

[58] A. Vinciarelli, "A Survey on Off-Line Cursive Word Recognition”, Pattern Recognition (PR), 35(7) : 1443-1446, 2002.

[59] M. Weindorf, "Structure Based Interpretation of Unstructured Vector Maps", Graphics Recognition (GREC), 2001.

[60] H. Xue, "Building Skeletal Graphs for Structural Feature Extraction on Handwriting Images", International Conference on Document Analysis And Recognition (ICDAR), 2001.

[61] S. Yoon, G. Kim, Y. Choi, Y. Lee, "New Paradigm for Segmentation and Recognition", Graphics Recognition (GREC), 2001.

[62] J.J. Zou, H. Yan, "Vectorization of Cartoon Drawings", Visual Information, 2000. 